\title{
Aspectos éticos de la informática médica: principios de uso y usuario apro- piado de sistemas computacionales en la atención clínica
}

Fernando Suárez-Obando ${ }^{1}$, Adriana Ordóñez Vásquez ${ }^{2}$

Resumen: La Informática Médica (IM) estudia la intersección entre la tecnología computacional, la medicina y la influencia del uso de la historia clínica electrónica y los sistemas inteligentes de apoyo diagnóstico en la toma de decisiones clínicas. El uso inadecuado de la tecnología puede desviar los propósitos de la IM hacia su aprovechamiento impropio por terceros involucrados en la atención clínica, tales como administradores de salud o agentes aseguradores. Se plantea que los principios de "uso y usuario apropiado de la aplicaciones en IM" sean los fundamentos con los cuales se maneje adecuadamente la tecnología computacional en salud. El desarrollo de estos principios debe basarse en la evaluación de las propias aplicaciones, recalcando que ésta debe realizarse con las mismas consideraciones de otros tipos de intervenciones médicas o quirúrgicas.

Palabras clave: informática médica, ética, actitud hacia los computadores

\section{Ethical aspects of medical informatics: principles for use and appropriate user of computational systems in clinical health care}

\begin{abstract}
Medical Informatics (MI) studies the intersection among computer technology, medicine and the influence of electronic clinical history and the intelligent systems for diagnosis support in clinical decision making. The inadequate use of technology may divert the purposes of MI towards an inadequate use by third parties involved in clinical health care, such as health care managers or insurance agents. The principles for "use and appropriate user for MI applications" as base are proposed to manage suitably computational technology in health care. The development of these principles must be based in the evaluation of their applications, emphasizing that the evaluation must be carried out with the same considerations as other types of medical or surgical interventions.
\end{abstract}

Key Words: medical informatics, ethics, attitude towards computers

\section{Aspectos éticos da informática médica: princípios de uso e usuário apropriado de sistemas computacionais na atençáo clínica}

Resumo: A Informática Médica (IM) estuda a interseção entre a tecnologia computacional, a medicina e a influência do uso da história clínica eletrônica e os sistemas inteligentes de apoio diagnóstico na tomada de decisóes clínicas. O uso inadequado da tecnologia pode desviar os propósitos da IM para seu aproveitamento inadequado por terceiros envolvidos na atenção clínica, tais como administradores de saúde ou agentes de seguros. Propóe-se que os princípios de "uso e usuário apropriado das aplicaçóes em IM" sejam os fundamentos com os quais se manipule adequadamente a tecnologia computacional em saúde. O desenvolvimento destes princípios deve se basear na avaliação das próprias aplicaçôes, recalcando que esta se deve realizar com as mesmas consideraçôes de outros tipos de intervençốes médicas ou cirúrgicas.

Palavras-chave: informática médica, ética, atitude para com os computadores

\footnotetext{
${ }^{1}$ Department of Biomedical Informatics. University of Pitttsburgh. Pittsburgh, PA. USA

Correspondencia: fes15@pitt.edu

${ }^{2}$ Instituto de Genética Humana. Facultad de Medicina. Pontificia Universidad Javeriana. Bogotá D.C., Colombia
} 


\section{Introducción}

La Informática Médica (IM) es la disciplina que aplica las ciencias de la información en el contexto de la medicina(1). Sus herramientas incluyen computadores, software especializado, terminología médica formal y sistemas de comunicación que optimizan el uso de la información en las áreas de la salud.

La IM integra la información sanitaria a través de la Historia Clínica Electrónica (HCE), que permite ingresar efectivamente los datos del paciente, acceder a datos históricos y evaluar con celeridad pruebas de laboratorio y pruebas de seguimiento(2), métodos que facilitan la documentación eficiente de los procesos(3), disminuyen los errores de prescripción(4) y mejoran el registro y análisis de los datos clínicos $(5,6)$.

La introducción en la IM de sistemas basados en inteligencia artificial (IA) ha generado herramientas específicas de soporte en la toma de decisiones clínicas, tales como los medios expertos de apoyo diagnóstico (SEAD), que son sistemas de interpretación de grandes volúmenes de datos, con la capacidad de "aprender" de resultados históricos, de la inclusión de nuevos datos y de la modificación de las reglas de inferencia(7).

Los sistemas inteligentes aplicados a la IM analizan las variables que determinan los cuadros clínicos y establecen probabilidades diagnósticas y pronósticas. Con el incremento de la capacidad computacional su desarrollo es cada vez más relevante para la IM, integrando un mayor número de variables procedentes de datos de laboratorio, resultados de patología e imágenes diagnósticas, que junto con la historia clínica modelan los estadios clínicos de un paciente.

En América Latina el desarrollo de la IM y la introducción de la HCE en centros de atención sanitaria se encuentran en etapas tempranas de implementación(8), por tanto, los dilemas éticos relacionados con el uso de la informática pondrán a prueba la capacidad de los sistemas de salud para tomar decisiones en medio de la convivencia del criterio médico y los sistemas computacionales(9). El presente artículo explora diversos aspectos éticos relacionados con la IM, planteando que los principios de "uso y usuario apropiado de la aplicaciones en Informática Médica" son los pilares sobre los cuales se debe manejar adecuadamente la tecnología computacional en salud. El desarrollo de estos principios debe basarse en la evaluación de las aplicaciones, destacando que ésta debe tener las mismas consideraciones que tienen valoraciones de otros tipos de intervenciones médicas o quirúrgicas.

\section{Historia Clínica Electrónica y sistemas de apo- yo al diagnóstico clínico}

En las instituciones de salud con sistemas computacionales clínico-administrativos, el proceso de atención sanitaria se define bajo un sistema de captura de datos en el cual el médico consigna la historia clínica en formato electrónico y accede rápidamente a resultados de laboratorio, patología y radiología, mientras que el control de gastos, la administración de recursos y la facturación se resuelven aprovechando la automatización y codificación de los procesos y accediendo a la información clínica desde las áreas gerenciales(10).

La integración de la información médica con los requerimientos administrativos permite que la auditoría de los procesos clínicos sea más eficiente(11), lo que conlleva a que las decisiones clínicas sean valoradas tanto en función del beneficio del paciente como en función de los costos de la atención, la sostenibilidad del sistema de salud y la correspondencia entre la cobertura de la atención según diversas pólizas y la condición clínica del enfermo.

El conflicto entre el criterio clínico y los parámetros administrativos de sostenibilidad y contención de costos se amplifica cuando la información fluye rápidamente entre las dependencias hospitalarias y se intensifica ante la presencia de herramientas que brindan criterios de objetividad a la toma de decisiones, tal es el caso, por ejemplo, de los SEAD en la atención de paciente de cuidado crítico.

Los SEAD son sistemas de inferencia a través de las cuales el médico ingresa información específica del paciente, y un sistema de deducción - basado en IA - analiza la información referenciando diversas base de datos. Dependiendo del tipo 
de SEAD, el sistema genera una recomendación específica sobre el curso clínico a seguir o estima el riesgo de un desenlace $(12,13)$. Por ejemplo, al incorporar a la HCE un sistema de puntaje pronóstico de pacientes críticos, como el sistema $\mathrm{APACHE}^{3}$ en forma de SEAD, la aplicación informática analiza las condiciones pronósticas del paciente, compara pacientes actuales con pacientes previos, evalúa el desempeño de las unidades de cuidado intensivo en términos de mortalidad ajustada por severidad, compara el desempeño entre unidades de diferentes hospitales y establece los perfiles fisiológicos con mejores probabilidades de supervivencia. Los datos derivados del análisis del SEAD durante diferentes periodos de observación son de gran utilidad en la investigación clínica y en los procesos de evaluación de calidad en la atención de una unidad de cuidado intensivo(14).

Con un sistema de estas características se pueden suponer varias situaciones. Por ejemplo, que los médicos afinen, a través del sistema inteligente, la identificación de perfiles fisiológicos de pacientes con alto riesgo de morir en una unidad de cuidado crítico. Información que puede ser utilizada para establecer formas de mejorar la atención o que puede ser manejada como argumento para contener los gastos, negando el pago por la atención a subsecuentes pacientes con el mismo perfil.

Se puede argumentar que muchas decisiones clínicas son realizadas con subjetividad o con evidencia fragmentada, por tanto, sería preferible tomar decisiones con base en datos objetivos como los que se desprenden de un análisis estadísticoprobabilístico basado en IA. Por tanto, ni la sociedad ni la aseguradora deberían pagar por el cuidado crítico cuando existe evidencia que indica que este será fútil. Un SEAD podría usarse para respaldar esta posición, estimulando un sistema de salud en el que los profesionales y las entidades de salud competirían exclusivamente con base en la optimización de costos y desempeños(15).

En otro escenario, el SEAD podría utilizarse para evaluar y desafiar las decisiones médicas, situa-

\footnotetext{
3 APACHE: Acrónimo del inglés "Acute Physiology and Chronic Health Evaluation”. Sistema de clasificación clínica que determina la gravedad del paciente a través de la evaluación de diversas variables fisiológicas, expresando la intensidad de la enfermedad y el estado clínico del paciente.
}

ción que puede ser estudiada por la compañía aseguradora, que decidiría sobre la aprobación de reembolsos a la institución, sobre la base de lo predicho por el sistema. Si el sistema está en desacuerdo con el criterio médico, la compañía podría argumentar no pagar por un procedimiento que no estaba indicado según los criterios de eficiencia basados en el SEAD.

De otra parte, si las decisiones clínicas se basan exclusivamente en sistemas inteligentes de diagnóstico y predicción, el avance científico de la medicina se vería afectado, debido a que parte del progreso acontece cuando se detectan circunstancias en las cuales un paciente mejora a pesar de su condición crítica: las investigaciones sobre ese fenómeno conllevan a mejorar los tratamientos aun en los casos más difíciles. Negar la atención médica o quirúrgica con base exclusivamente en la herramienta predictiva y en puntos de corte de mortalidad predicha, conllevaría a un estancamiento del progreso, dado que al alcanzar un puntaje específico de gravedad todos los pacientes morirían a consecuencia de la enfermedad y de la suspensión de los tratamientos, convirtiéndose el manejo clínico en una profecía autocumplida, que dejaría de lado la posibilidad de salvar al enfermo siempre que este tenga un alto riesgo de mortalidad predicho por el puntaje.

En los ejemplos descritos, el sistema de predicción puede ser utilizado para justificar la suspensión del tratamiento y la contención del gasto, ignorando el criterio médico en favor de un juicio derivado del sistema inteligente. Al mismo tiempo, la aplicación puede ser utilizada para detectar situaciones clínicamente complejas, que merecerían el desarrollo de medidas terapéuticas que conlleven a tratar pacientes críticamente enfermos.

Las posiciones contradictorias entre la optimización del gasto, el criterio médico y el desarrollo científico de la medicina, en razón de la presencia de un sistema que pretende convertir variables clínicas en elementos objetivos para la toma de decisiones, deben buscar un equilibrio en el que la aplicación persiga, en primer lugar, el beneficio del paciente. Si el uso adecuado de las herramientas de la informática médica deben partir del compromiso del médico con el enfermo, el criterio médico debe encaminar el uso de herramien- 
tas informáticas hacia su aprovechamiento como herramienta clínica complementaria, y no como un sistema de corte administrativo que clasifique unilateralmente el futuro del paciente.

Sin embargo, el juicio clínico frente al uso del computador requiere de otros argumentos que demuestren que la aplicación de la informática en la atención clínica es mucho más que una tendencia tecnológica y mucho menos que el remplazo del criterio del médico. Esos argumentos deben buscar el equilibrio entre el criterio que busca lo mejor para el paciente y los aspectos administrativos que inevitablemente son parte de la práctica médica.

El uso apropiado de los computadores por usuarios propicios que valoren la privacidad y confidencialidad del paciente son los pilares fundamentales del manejo ético de las herramientas de la IM, y sobre estos elementos se funda el equilibrio entre el manejo clínico, el progreso a través de la investigación y el uso adecuado de los recursos en presencia de la tecnología computacional.

\section{Uso apropiado de las aplicaciones en Informá- tica Médica}

La introducción de computadores en el ambiente clínico inicialmente se acompañó de grandes expectativas; célebres sistemas de finales del siglo XX, como el MYCIN ${ }^{4}(16)$ y el INTERNIST$\mathrm{I}^{5}(17)$, presagiaban el desarrollo de medios que equipararían al médico en la toma de decisiones. Sin embargo, la complejidad del conocimiento médico definió claramente que los sistemas de apoyo diagnóstico son apropiados, especialmente a través del conocimiento de sus limitaciones, entendiendo que el computador no abroga el juicio clínico del médico frente a las recomendaciones derivadas de sistemas inteligentes.

Las habilidades requeridas para el diagnóstico y manejo de la enfermedad difieren de la capacidad de un programa para analizar datos, por tanto el uso de computadores en la clínica se limita a aspectos concretos, como la disminución de errores y el mejoramiento de estándares(18). El principio que ahora exponemos se denomina: "uso apropiado de la aplicaciones en Informática Médica” (19),

\footnotetext{
${ }^{4}$ Sistema computarizado de selección de terapia antimicrobiana.

${ }^{5}$ Sistema experto de diagnóstico de enfermedades complejas.
}

al cual adhieren tanto desarrolladores de software como médicos o administradores de sistemas, y establece el uso y curso apropiado de la tecnología como adyuvante y no como decisor. Del uso apropiado se desprende que los propósitos clínicos del sistema no deben ser utilizados como elementos a partir de los cuales terceros involucrados tomen decisiones sobre el desembolso de recursos, el precio de las pólizas o la clasificación y selección de pacientes.

El uso apropiado del sistema computacional corresponde a los objetivos de la aplicación y no a otras tareas impuestas durante su uso. Los objetivos de un sistema de apoyo diagnóstico tienen como propósito clínico la disminución de errores, dado que presenta los datos con mayor exactitud en los momentos adecuados de la atención. Si los datos obtenidos son considerados para la investigación clínica, se incrementa la calidad de los estándares de manejo con base en resultados derivados de un análisis científico. En ambos casos, la disminución de errores y el mejoramiento de la atención basado en la investigación señalan que el principio del uso adecuado de las aplicaciones es, en esencia, un dominio clínico y no administrativo.

Este principio también señala que, aun cuando los datos derivados de una aplicación informática sean utilizados en el análisis de estrategias relacionadas con la contención del gasto, el límite de su uso es el del ámbito clínico, y el objetivo de la aplicación no se extiende hasta justificar un poder decisorio de las aseguradoras o del personal administrativo.

El principio del uso adecuado se construye desde las tempranas etapas de diseño de la aplicación, se fortalece durante su desarrollo y se perfecciona durante la implementación del mismo en el ámbito hospitalario. En el caso de los sistemas informáticos, el proceso de desarrollo se basa en diversas formas de evaluación. Según el resultado de las evaluaciones, el sistema se modifica hasta convertirse en una aplicación útil al usuario, quien, a su vez, evaluará el sistema dentro del contexto clínico.

Sin embargo, antes de pasar a la especificidad de la evaluación se debe recalcar que la información 
originada en el análisis de variables por un sistema computacional puede ser utilizada de formas diferentes a las proyectadas. Incluso un sistema seguro seguirá dejando en la vulnerabilidad los datos que emerjan después de los procesos de inferencia si los usuarios no mantienen la responsabilidad del uso apropiado de los computadores.

\section{Usuario apropiado de las aplicaciones en In- formática Médica}

El uso eficiente del SEAD requiere entrenamiento, experiencia y educación por parte del usuario, características que definen una conducta de aprovechamiento de la tecnología, en la cual el médico utiliza la aplicación computacional como un referente que se confronta con los objetivos diagnósticos y terapéuticos, y se contrasta con las prácticas clínicas aceptadas para alcanzar esos objetivos, incluyendo el reconocimiento de las características y requerimientos individuales del paciente.

La experiencia clínica y el conocimiento de la aplicación computacional permiten al clínico interpretar la información arrojada por el sistema y aplicarla en el momento adecuado. De igual forma, le permiten reconocer los errores del sistema y decidir cuándo omitir información inconveniente o irrelevante. Estas características definen al "usuario apropiado de la aplicaciones en Informática Médica”.

El usuario apropiado debe conjugar el conocimiento profundo de la medicina y la experiencia clínica con el conocimiento de la aplicación y sus objetivos. Para cumplir estos requerimientos se requiere entrenamiento y comprensión de los fundamentos informáticos.

La noción de un usuario apropiado empoderado del sistema apropiado encamina las ayudas computacionales a cumplir objetivos clínicos; sin embargo, la garantía de la seguridad de los datos del paciente y de su condición médica dependen de elementos de seguridad que pueden salirse del control del usuario y que no necesariamente dependen del diseño del sistema computacional. En la protección de la confidencialidad y privacidad de la historia clínica se encuentra el equilibrio entre el acceso a la información, el cumplimiento de los objetivos clínicos de una aplicación y el protagonismo del usuario adecuado en la protección de los datos clínicos.

\section{Confidencialidad y privacidad}

La principal ventaja del uso de los computadores en medicina es el acceso rápido a la información; sin embargo, la facilidad para localizar datos es al mismo tiempo su máxima debilidad. En principio, la información clínica solo debe estar disponible para aquellos interesados en el cuidado médico del paciente; no obstante, la información puede estar a disposición de personal de salud que no la necesita, o abierta a terceros que pueden usarla para infringir daño físico, emocional o financiero al paciente. Por tanto, a los objetivos clínicos de un sistema se debe añadir características de seguridad que permitan garantizar el mejoramiento de la atención, accediendo a la información oportunamente y protegiendo la confidencialidad a través de la restricción del acceso(20).

No es posible construir un sistema completamente seguro; solo es posible minimizar su vulnerabilidad maximizando las restricciones de acceso. La responsabilidad de mantener la seguridad del sistema recae tanto en los diseñadores y sus administradores como en los usuarios: médicos, enfermeras u otro personal hospitalario.

El uso adecuado de un sistema de predicción o de diagnóstico asistido por computador depende del cumplimiento de sus objetivos clínicos, es decir, de la interpretación correcta de los datos y del reconocimiento de sus límites en la toma de decisiones, esta acción, ejercida por el usuario apropiado (personal autorizado y entrenado) en conjunto con el cumplimiento de elevados estándares de seguridad, mantiene la privacidad y confidencialidad del paciente(21).

Sin embargo, la armonía entre sistema, usuario y seguridad no se define en el momento de la implementación o uso de los computadores. La naturaleza flexible y cambiante del ejercicio clínico indica que debe ejercerse una observación estricta de las aplicaciones computacionales antes de su implementación y durante su utilización en la práctica clínica. Esta observación estricta es la base de la evaluación. 


\section{La evaluación de aplicaciones en Informática Médica}

La Informática Médica no es un área de estudio que considere exclusivamente los computadores en la medicina, si no que se construye sobre la introducción de herramientas tecnológicas en ambientes clínicos con prácticas sociales establecidas(22), por tanto, los efectos de la computarización de procesos clínicos deben ser sujeto de análisis en relación con las consecuencias sociales y profesionales de su implementación.

El termino "evaluación" describe un amplio rango de actividades de recolección de datos utilizados en la implementación de métodos de análisis y valoración de la tecnología computacional. La evaluación se basa en metodología científica que mide el efecto de los computadores en la práctica clínica, utilizando los resultados de la valoración en el mejoramiento del cuidado de la salud a través de la optimización de los procesos.

Se debe diferenciar la evaluación en IM de la evaluación de tecnologías sanitarias. Esta última es un concepto general que mide las consecuencias clínicas, sociales, éticas y económicas de la tecnología en salud, por tanto, abarca temas tan diversos como la eficacia de la Tomografía por Emisión de Positrones(23) o la eficiencia de la inmuno-profilaxis en prematuros(24), mientras que la evaluación en IM enfoca sus objetivos a la valoración de las consecuencias clínicas, éticas, sociales y económicas del uso de computadores e informática en medicina.

La evaluación de una aplicación de informática médica se fundamenta en cuestionamientos encaminados a determinar la necesidad de la aplicación informática, su confiabilidad, seguridad y su impacto en los usuarios y el paciente. Los cuestionamientos sobre la aplicación informática cambian a medida que progresa la implementación, y la medición del impacto sobre la salud del paciente o la generalización del sistema a otros hospitales dependen del impacto medido en las etapas tempranas de desarrollo, requiriendo tiempos prolongados de observación. Por tanto, las mediciones de variables clínicas que son parte de evaluaciones de eficiencia y costo beneficio deben ser monitorizadas continuamente y los usuarios comprender la importancia de dichas evaluaciones con el fin de detectar fallas y fortalezas de la aplicación.

La implementación de un sistema de evaluación surge de la necesidad de identificar el sistema apropiado y verificar el cumplimiento de sus objetivos clínicos, caracterizar al usuario apropiado y evaluar su desempeńo, comprobando el cumplimiento de los objetivos de seguridad e identificando quienes deben tener acceso a la información. Cualquier acción encaminada a establecer prácticas éticas en la informática médica debe incluir procedimientos de evaluación que, más allá de un control de calidad, definen las obligaciones de los desarrolladores, usuarios y administradores.

En el escenario del SEAD, los objetivos clínicos se establecen desde el diseño del software: al determinar que las mediciones fisiológicas se integran a la HCE, se diseñan alertas en la aplicación informática que, ante valores extremos, se hacen visibles al médico brindando opciones de manejo, de tal modo que el médico tiene acceso rápido a la información y está bajo su criterio clínico la toma de decisiones subsiguientes. Sin embargo, el cumplimiento de los objetivos del SEAD ha de verificarse en su uso cotidiano; por ejemplo, una evaluación enfocada a medir el desempeńo del sistema de predicción de mortalidad, en relación con el cumplimiento de protocolos de manejo, brindará información sobre la correlación entre las alertas de valores extremos y la aplicación de los datos derivados del SEAD. Si el protocolo de evaluación comprende el análisis del cumplimiento de los objetivos clínicos, debe ser capaz de detectar si los datos están siendo aplicados o no y bajo cuáles circunstancias, de tal forma que el resultado de la evaluación puede indicar incluso que el SEAD no aporta ningún beneficio en el cuidado del paciente y que, por el contrario, satura de información al médico sin que el enfermo se beneficie de los datos derivados de la herramienta tecnológica.

Si la evaluación solo se lleva a cabo en los procesos de diseño e implementación temprana, no será posible verificar si sus objetivos se cumplen en la práctica, aun cuando la necesidad del SEAD haya sido bien justificada. 
La evaluación puede detectar que los aspectos relacionados con la estructura jerárquica y social de una unidad hospitalaria son los que comprometen el cumplimiento de los objetivos clínicos, aun cuando el sistema computacional en sí mismo no tenga fallas en su diseño(25). La evaluación puede detectar fallas en el diseño al descubrir situaciones en las cuales los protocolos de seguridad del sistema no se cumplen por dificultades de visualización e interacción entre el computador y el usuario, comprometiendo la confidencialidad y privacidad de la historia clínica $(26,27)$.

Si los resultados de la evaluación de un sistema determinan que el uso del computador no corresponde a los objetivos de la aplicación, si no a otras tareas impuestas durante su uso cotidiano, la evaluación estará detectando el incumplimiento del uso apropiado de los computadores; en el momento en que la evaluación revele que quienes acceden al sistema no tienen entrenamiento, experiencia o conocimiento, estará detectando el acceso de usuarios inapropiados que comprometen los objetivos y seguridad de los datos.

Cuando un hospital decide sistematizar todos sus procesos administrativos, implementar la prescripción electrónica de medicamentos y desarrollar un sistema integrado de historia clínica, laboratorio y radiología, puede crear su propio software o comprarlo en el mercado de tecnologías. De cualquier modo, debe tener en cuenta que los procesos de evaluación deben integrar el proceso de sistematización y que la medición del impacto de la tecnología en el paciente y el personal de salud forman parte de las mismas actividades de vigilancia que se hacen sobre cualquier otro tipo de tecnología médica. Así como se evalúan los eventos adversos de un medicamento recientemente introducido al mercado, se debe evaluar el impacto tanto de la introducción de computadores en la atención clínica como de su continua presencia en casi todos los procesos clínicos.

No debe darse por hecho que el uso de tecnología y la sistematización de la atención signifiquen necesariamente un avance, ni ha de suponerse que el uso de computadores o la digitalización de procesos es garantía de progreso. Varias experiencias han demostrado que sin monitorización constante y en ausencia de la evaluación apropia- da, la vida y seguridad del paciente pueden verse gravemente comprometidas en presencia de sistemas computacionales previamente considerados seguros(28-30). Aún más, la introducción de computadores en un hospital genera cambios en la estructura organizacional(31), ocasiona estrés relacionado con el aprendizaje de nuevas tecnologías(32) y puede conllevar a una sobrecarga de trabajo para el personal de salud(33). Si se dan por hecho los beneficios, los efectos negativos de la tecnología se detectarán tardíamente y si los beneficios de los computadores no son medidos en el contexto de su desarrollo clínico, no será posible conocer la magnitud ni el sentido del impacto de la tecnología en la salud de las personas.

La Informática Médica es una disciplina joven, muchos de los postulados teóricos sobre los beneficios de la salud electrónica están lejos de ser verificados y su costo-efectividad aun tiene que ser demostrada en la práctica(34). De los resultados de la evaluación depende la continuidad, modificación o suspensión de un sistema informático, por tanto la evaluación de las aplicaciones de la IM es el pilar fundamental sobre el cual recae la demostración de sus errores o de sus virtudes.

La evaluación de los cambios suscitados después de la implementación de la HCE, en un hospital, o de un SEAD, en una unidad de cuidado crítico, permite definir las condiciones básicas con las cuales un sistema de salud ha trabajado tradicionalmente y establece el impacto que la tecnología computacional tiene en la atención en salud al modificar las condiciones previas(35). La evaluación brinda a médicos y desarrolladores la posibilidad de establecer las circunstancias a partir de las cuales se generan tendencias de mortalidad y seguridad de los pacientes ante la sistematización de la atención clínica $(36,37)$. La evaluación permite descubrir falencias de diseño que comprometen la seguridad de los datos(38) y analizar la prioridad de implementar nuevos servicios y tecnologías según las necesidades de la institución(39).

De acuerdo con los estándares actuales, la introducción de un medicamento o la aplicación de una intervención terapéutica novedosa se lleva a cabo si el proceso se acompaña de investigación y evaluación científica apropiadas, lo que es especialmente riguroso en el caso de los productos 
de la industria farmacéutica. De igual forma, la medicina basada en la evidencia y la epidemiologia clínica han establecido parámetros formales para evaluar y calificar las intervenciones médicas. La evidencia que soporta el empleo de una intervención se recolecta desde tempranas etapas del proceso investigativo, como puede ser la fase I de los estudios farmacológicos, hasta los estudios post-mercadeo de un medicamento. Esta misma rigurosidad se aplica a la introducción de nuevos dispositivos médicos, sean estos un catéter o un nuevo material de osteosíntesis. La rigurosidad y la metodología evaluativa han de ser implementadas en las aplicaciones de la Informática Médica, dado que, al igual que un medicamento, un dispositivo médico o cualquier otra forma de intervención médica, las aplicaciones forman parte de tecnologías que contribuyen directa o indirectamente a la toma de decisiones y al desarrollo de desenlaces clínicos.
En América Latina el desarrollo de sistemas informáticos en hospitales y centros de salud comienza a vislumbrarse como un programa de investigación relevante para el desarrollo de la región $(40,41)$, lo que constituye un momento adecuado para que, en conjunto con el desarrollo de la Informática Médica, los sistemas de salud implementen sistemas de evaluación encaminados a identificar y verificar tanto el "uso y usuario apropiado de las aplicaciones en Informática Médica" como la confidencialidad de la información y la confiabilidad de las aplicaciones.

\section{Agradecimientos}

Este trabajo fue posible por la colaboración entre la Pontificia Universidad Javeriana y The Department of Biomedical Informatics, University of Pitttsburgh. Trabajo parcialmente patrocinado por Fogarty International Center NIH Grant No 1 D43 TW008443-01

\section{Referencias}

1. Bernstam EV, Smith JW, Johnson TR. What is biomedical informatics? J Biomed Inform 2010; 43(1): 104-110.

2. Main C, et al. Computerised decision support systems in order communication for diagnostic, screening or monitoring test ordering: systematic reviews of the effects and cost-effectiveness of systems. Health Technol Assess 2010; 14(48): 1-227.

3. Kelley TF, Brandon DH, Docherty SL. Electronic nursing documentation as a strategy to improve quality of patient care. J Nurs Scholarsh 2011; 43(2): 154-162.

4. Zlabek JA, Wickus JW, Mathiason MA. Early cost and safety benefits of an inpatient electronic health record. J Am Med Inform Assoc 2011; 18(2): 169-172.

5. Shekelle PG, Morton SC, Keeler EB. Costs and benefits of health information technology. Evid Rep Technol Assess (Full Rep) 2006; 132: 1-71.

6. Hayrinen K, Saranto K, Nykanen P. Definition, structure, content, use and impacts of electronic health records: a review of the research literature. Int J Med Inform 2008; 77(5): 291-304.

7. Basheer IA, Hajmeer M. Artificial neural networks: fundamentals, computing, design, and application. J Microbiol Methods 2000; 43(1): 3-31.

8. Rodrigues RJ. Risk A. eHealth in Latin America and the Caribbean: development and policy issues. J Med Internet Res 2003; 5(1): e4.

9. Goodman KW. Ethics and Health Informatics: focus on Latin America and the Caribean. Acta Bioethica 2005; 11(2): 121-126.

10. Karsh BT, et al. Health information technology: fallacies and sober realities. J Am Med Inform Assoc 2010; 17(6): 617-623.

11. Bowes WA. 3rd, Measuring use of electronic health record functionality using system audit information. Stud Health Technol Inform 2010; 160(Pt 1): 86-90.

12. Ramesh AN, et al. Artificial intelligence in medicine. Ann R Coll Surg Engl 2004; 86(5): 334-338.

13. Pandey B, Mishra RB. Knowledge and intelligent computing system in medicine. Comput Biol Med 2009; 39(3): 215-230. 
14. Gartman EJ, et al. Using serial severity scores to predict death in ICU patients: a validation study and review of the literature. Curr Opin Crit Care 2009; 15(6): 578-582.

15. Hodgman SB. Predictive modeling \& outcomes. Prof Case Manag 2008; 13(1): 19-23.

16. Wraith SM, et al. Computerized consultation system for selection of antimicrobial therapy. Am J Hosp Pharm 1976; 33(12): 1304-1308.

17. Miller RA. A History of the INTERNIST-1 and Quick Medical Reference (QMR) ComputerAssisted Diagnosis Projects, with Lessons Learned. Yearb Med Inform 2010; 121-36; 0943-4747.

18. Manning RC. Why Sherlock Holmes can't be replaced by an expert system. Philosophical Studies 1987; 51(January): 19-28.

19. Miller RA. Why the standard view is standard: people, not machines, understand patients' problems. J Med Philos 1990; 15(6): 581-591.

20. Haas S, et al. Aspects of privacy for electronic health records. Int J Med Inform 2011; 80(2): 26-31.

21. Meingast M, Roosta T, Sastry S. Security and privacy issues with health care information technology. Conf Proc IEEE Eng Med Biol Soc 2006; 1: 5453-8.

22. Peute LW, et al. Anatomy of a failure: a sociotechnical evaluation of a laboratory physician order entry system implementation. Int J Med Inform 2010; 79(4): 58-70.

23. Cooper KL, et al. Positron emission tomography (PET) and magnetic resonance imaging (MRI) for the assessment of axillary lymph node metastases in early breast cancer: systematic review and economic evaluation. Health Technol Assess 2011; 15(4): 1-134.

24. Wang D, Bayliss S, Meads C. Palivizumab for immunoprophylaxis of respiratory syncytial virus (RSV) bronchiolitis in high-risk infants and young children: a systematic review and additional economic modelling of subgroup analyses. Health Technol Assess 2011; 15(5): 1-124.

25. Bartos CE, et al. Negative CPOE attitudes correlate with diminished power in the workplace. AMIA Annu Symp Proc 2008: 36-40.

26. Malin B, Airoldi E. Confidentiality preserving audits of electronic medical record access. Stud Health Technol Inform 2007; 129(Pt 1): 320-324.

27. Wilcox AB, Chen YH, Hripcsak G. Minimizing electronic health record patient-note mismatches. J Am Med Inform Assoc 2011.

28. Israelski EW, Muto WH. Human factors risk management as a way to improve medical device safety: a case study of the therac 25 radiation therapy system. Jt Comm J Qual Saf 2004; 30(12): 689-695.

29. Weir CR, et al. Direct text entry in electronic progress notes. An evaluation of input errors. Methods Inf Med 2003; 42(1): 61-67.

30. Khajouei R, Jaspers MW. The impact of CPOE medication systems' design aspects on usability, workflow and medication orders: a systematic review. Methods Inf Med 2010; 49(1): 3-19.

31. Morrison J, Lindberg P. When no one has time: measuring the impact of computerization on health care workers. AAOHN J 2008; 56(9): 373-378.

32. Griffiths KL, Mackey MG, Adamson BJ. The impact of a computerized work environment on professional occupational groups and behavioural and physiological risk factors for musculoskeletal symptoms: a literature review. J Occup Rehabil 2007; 17(4): 743-765.

33. Podichetty V, Penn D. The progressive roles of electronic medicine: benefits, concerns, and costs. Am J Med Sci 2004; 328(2): 94-99.

34. Black AD, et al. The impact of eHealth on the quality and safety of health care: a systematic overview. PLoS Med 2011; 8(1): e1000387.

35. Li J. A Sociotechnical Approach to Evaluating the Impact of ICT on Clinical Care Environments. Open Med Inform J 2010; 4: 202-205.

36. Yu F, et al. The relationship between computerized physician order entry and pediatric adverse drug events: a nested matched case-control study. Pharmacoepidemiol Drug Saf 2009; 18(8): 751755.

37. Han YY, et al. Unexpected increased mortality after implementation of a commercially sold computerized physician order entry system. Pediatrics 2005; 116(6): 1506-1512. 
38. Gomes R, Lapao LV. The adoption of IT security standards in a healthcare environment. Stud Health Technol Inform 2008; 136: 765-770.

39. Blumenthal D, Tavenner M. The "meaningful use" regulation for electronic health records. NEngl J Med 2010; 363(6): 501-504.

40. Curioso WH, et al. Strengthening global health informatics research within the andean region through international collaboration. Rev Peru Med Exp Salud Publica 2010; 27(3): 449-457.

41. Blaya JA, et al. Full impact of laboratory information system requires direct use by clinical staff: cluster randomized controlled trial. J Am Med Inform Assoc 2011; 18(1): 11-16.

Recibido: 12 de septiembre de 2011

Aceptado: 18 de octubre de 2011 